\title{
Cardiac Safety of (Neo)Adjuvant Trastuzumab in the Community Setting: A Single-Center Experience
}

\author{
Leonardo Gomes da Fonseca Debora de Melo Gagliato Tiago K. Takahashi \\ Milena Perez Mak Romualdo Barroso-Sousa Laura Testa Vanessa Petry Helena \\ Romulo de Paula Costa Paulo M. Hoff Max S. Mano
}

Division of Medical Oncology, Instituto do Câncer do Estado de São Paulo, Faculdade de Medicina, Universidade de São Paulo, São Paulo, Brazil

\section{Keywords}

Adjuvant therapy - Breast cancer - Cardiotoxicity .

Trastuzumab

\section{Summary}

Background: Trastuzumab improves the survival of patients with human epidermal growth factor receptor 2 (HER2)-positive breast cancer (BC). The incidence and long-term impact of trastuzumab-related cardiotoxicity in the community setting is of great clinical importance. Material and Methods: Patients with HER2-positive BC treated with (neo)adjuvant trastuzumab were retrospectively evaluated. Cardiotoxicity was defined as cardiac death or absolute decrease in left ventricular ejection fraction of at least $10 \%$ to a value less than $50 \%$, or symptomatic heart failure. Results: We evaluated 237 patients: median age 53 years (range $27-83$ years). $40.5 \%$ of these patients had received neoadjuvant and $59.5 \%$ adjuvant chemotherapy. The majority (83.9\%) were treated with an anthracycline-based regimen. Median exposure to trastuzumab was 8 months (range 2-12 months). Cardiotoxicity was diagnosed in $20.2 \%$, but symptoms only occurred in $3.8 \% .41 .6 \%$ recovered cardiac function. None of the risk factors were associated with cardiotoxicity. Conclusion: The incidence of trastuzumab-related cardiotoxicity found in this study was slightly higher than those reported in randomized clinical trials. Nevertheless, most patients were asymptomatic. We describe the cardiac outcomes of a non-selected population, which possibly reflects those found in the 'real world'. The risks versus benefits of trastuzumab use remain in favor of treatment, but cardiotoxicity should be monitored.

\section{Introduction}

Breast cancer (BC) is the most common cancer among females and the most common cause of cancer death in women [1]. Approximately $15-20 \%$ of BC patients have gene amplification or overexpression of human epidermal growth factor receptor 2 (HER2) [2, 3].

Trastuzumab is a monoclonal antibody that targets HER2. Therapy with trastuzumab benefits patients whose tumors have overexpression or amplification of HER2. Major clinical trials have demonstrated that this drug improves disease-free survival and overall survival in the adjuvant and metastatic setting [4-9]. Furthermore, improvement in response rate and complete pathological response was demonstrated when trastuzumab was added to chemotherapy in the neoadjuvant setting [10-12].

However, cardiac dysfunction may be a potentially serious side effect associated with this agent $[13,14]$. It is frequently manifested by an asymptomatic decrease in left ventricular ejection fraction (LVEF) and less commonly by congestive heart failure. The mechanism behind this event is not fully understood. Evidence from both in vivo and in vitro studies indicates that HER2 may play a critical role in the development of the embryonic heart. Deletion of this gene in cultured rat cardiomyocytes leads to changes that may influence the cell cycle [15]. In an adult heart, HER2 may continue to have an important function in modifying the cardiac response to stress [16-18].

The risk of trastuzumab-related cardiotoxicity seems to be associated with older age, higher body mass index, hypertension and use of anthracyclines [19-23]. The first clinically detected evidence of cardiac damage with trastuzumab came from a phase III trial in the metastatic setting. Trastuzumab

\section{KARGER \\ Fax +497614520714}

Information@Karger.com

www.karger.com
(C) 2014 S. Karger GmbH, Freiburg

$1661-3791 / 14 / 0094-0255 \$ 39.50 / 0$

Accessible online at:

www.karger.com/brc
Dr. Leonardo Gomes da Fonseca

Division of Medical Oncology

Instituto do Câncer do Estado de São Paulo, Faculdade de Medicina, Universidade de São Paulo

Av. Dr. Arnaldo 251, São Paulo São Paulo, Brazil

leogfonseca@hotmail.com 
administered concomitantly with paclitaxel was associated with cardiac dysfunction in $13 \%$ of patients and together with anthracycline and cyclophosphamide with $27 \%$ of patients. In contrast, the incidence of cardiotoxicity in patients who did not received trastuzumab was $8 \%$ and $1 \%$ when treated with anthracycline plus cyclophosphamide and paclitaxel, respectively [9]. Trastuzumab alone was associated with cardiotoxicity rates of $3-7 \%$ in a retrospective review of patients enrolled in initial phase II and III trials [13].

Subsequently, trials evaluating trastuzumab in the adjuvant setting were required to provide more stringent and consistent cardiac monitoring, and to exclude patients with abnormal cardiac function or cardiovascular risk factors. As a consequence, the incidence of trastuzumab-associated cardiotoxicity was lower in later trials $[4,5,7,8]$.

The risk of cardiac dysfunction in the general population may be even greater than those described in selected patients from clinical trials. The widespread use of trastuzumab in the community setting often results in a safety profile that does not always mirror that reported in prospective randomized trials [4-9, 24, 25].

Since 2008, trastuzumab has been available within the Brazilian public health system. We hypothesized that cardiac toxicity might be somewhat different in a non-selected patient population compared to that found in controlled clinical trials. We therefore conducted a retrospective study to report our single-center experience with respect to the cardiac safety of this drug and to identify possible risk factors for cardiac events.

\section{Patients and Methods}

We performed a retrospective review of the cohort of patients with HER2-positive BC treated at the Instituto do Câncer do Estado de São Paulo, Faculdade de Medicina da Universidade de São Paulo, Brazil. We included patients treated between July 2008 and October 2012. None of these patients had been treated in the context of clinical trials.

Patients were eligible for the study if they had: (1) histologically confirmed BC, (2) non-metastatic disease, (3) HER2 overexpression or amplification assessed by immunohistochemical analysis with a score of $3+$ or positive fluorescence in situ hybridization (FISH), (4) received trastuzumab in our institution with or without chemotherapy in the adjuvant or neoadjuvant setting, (5) received at least 1 infusion of trastuzumab in our institution, or (6) LVEF assessments carried out while undergoing treatment. This present analysis was approved by the local ethics committee.

\section{Assessment and Outcomes}

We identified our patient cohort using the institutional medical records database. Data abstracted from patients' files included: age, sex, body mass index, performance status, histological subtype, hormone receptor status, TNM staging, hypertension (defined as blood pressure $\geq 140 / 90 \mathrm{mmHg}$ or use of antihypertensive drugs), occurrence of diabetes mellitus, and previous cardiopathy - defined by a previous diagnosis of heart failure, LVEF below the lower limit of normal, previous ischemic cardiopathy (Q-wave or ischemic ST segment on electrocardiogram and/ or echocardiographic segmental wall motion abnormalities), angina pectoris, valvular disease (cardiac murmur with echocardiographic evidence of moderate to severe valvular regurgitation and/or stenosis of any grade), and arrhythmias requiring therapy. Information about treatment was also collected, such as chemotherapy schedule, use and cumulative dose of anthracyclines, radiotherapy treatment, and number of trastuzumab cycles as well as drug interruption. According to our institute protocol, as part of the routine pre-chemotherapy evaluation, all patients should undergo a baseline cardiac examination and echocardiography. These examinations should be repeated every 3 months as long as therapy continues. Cardiotoxicity was analyzed by LVEF determined by echocardiography or by the presence of symptoms of cardiac failure. For LVEF measurements, the quantitative Teicholz method was used, and the lower limit of LVEF considered was 55\%. Baseline and follow-up echocardiographies were performed at our institution using the same method. The definition of cardiotoxicity was: (1) cardiac death, (2) functional class III or IV according to New York Heart Association (NYHA) system, or (3) an LVEF of $\leq 50 \%$ with an absolute decrease of $\geq 10 \%$ from baseline. The decision to interrupt trastuzumab was made on the judgment of an oncologist. Reversibility was defined as: (1) recovery of the LVEF to at least the baseline value, or (2) remission of congestive symptoms with or without use of diuretics, beta-blockers, angiotensin II receptor blockers or angiotensin-converting enzyme (ACE) inhibitor.

For patients who had experienced a cardiac event, the dates of any temporary or definitive suspension of trastuzumab, and data on cardiac medications prescribed, recovery of LVEF and hospital admission for heart failure were obtained.

\section{Statistical Analysis}

Descriptive statistics were used to describe demographics and baseline characteristics. The association between patients who experienced a cardiac event and risk factors was analyzed by a chi-squared test. All analyzes were performed using SPSS software 11.0. (SPSS Chicago, IL, USA). Statistical significance for all tests was defined as $\mathrm{p}<0.05$.

\section{Results}

\section{Patient Characteristics}

A total of 237 patients met all inclusion criteria; for 16 additional patients, although they met the all other inclusion criteria, LVEF assessment data were not present in their medical records and they were consequently not included in the analysis. Baseline characteristics are listed in table 1.

Median age at the start of trastuzumab therapy was 53 years (range $27-83$ years). Most patients had stage II or III disease (22.3 and $37.1 \%$, respectively) and $63.7 \%$ had estrogen receptor (ER)-positive tumors. 72 patients $(30.4 \%)$ had hypertension, $21(8.8 \%)$ diabetes mellitus and 58 patients $(24.5 \%)$ were past or active smokers. Previous cardiopathy was found in 14 patients $(5.9 \%)$, including 7 with ischemic cardiomyopathy $(2.9 \%), 3(1.2 \%)$ with arrhythmias requiring therapy, $2(0.8 \%)$ with valvular disease due to rheumatic valve disease, $1(0.4 \%)$ with chagasic cardiomyopathy and 1 with hypertrophic cardiomyopathy (0.4\%). 59 patients $(21.6 \%)$ had been treated for hypertension with an antihypertensive drug.

The majority of patients $(83.9 \%)$ were treated with an anthracycline-based regimen, sequentially to trastuzumab. The anthracycline currently adopted in our institution is doxorubicin. The mean cumulative dose of doxorubicin was $233.9 \mathrm{mg} /$ 
Table 1. Baseline characteristics of patients

\begin{tabular}{|c|c|}
\hline Patients & \\
\hline Total, n (\%) & $237(100 \%)$ \\
\hline Median age, years (range) & $53(27-83)$ \\
\hline Median body mass index, $\mathrm{kg} / \mathrm{m}^{2}$ (range) & $27.4(17-46)$ \\
\hline \multicolumn{2}{|l|}{ ECOG performance status, n (\%) } \\
\hline 0 & $227(95.8 \%)$ \\
\hline 1 & $9(3.8 \%)$ \\
\hline 2 & $1(0.4 \%)$ \\
\hline \multicolumn{2}{|l|}{ Hypertension, n (\%) } \\
\hline Yes & $72(30.5 \%)$ \\
\hline No & $165(69.5 \%)$ \\
\hline \multicolumn{2}{|l|}{ Diabetes, n (\%) } \\
\hline Yes & $21(8.9 \%)$ \\
\hline No & $216(91.1 \%)$ \\
\hline \multicolumn{2}{|l|}{ Cardiopathy, n (\%) } \\
\hline Yes & $14(5.9 \%)$ \\
\hline No & $223(94.1 \%)$ \\
\hline \multicolumn{2}{|l|}{ Histology, n (\%) } \\
\hline Ductal & $235(99.2 \%)$ \\
\hline Others & $2(0.8 \%)$ \\
\hline \multicolumn{2}{|l|}{ Stage, $\mathrm{n}(\%)$} \\
\hline $\mathrm{I}$ & $53(22.3 \%)$ \\
\hline II & $96(40.5 \%)$ \\
\hline III & $88(37.1 \%)$ \\
\hline \multicolumn{2}{|l|}{ Hormone receptor status, $\mathrm{n}(\%)$} \\
\hline $\mathrm{E}+\mathrm{P}+$ & $130(54.8 \%)$ \\
\hline E-P- & $84(35.4 \%)$ \\
\hline $\mathrm{E}+\mathrm{P}-$ & $21(8.9 \%)$ \\
\hline $\mathrm{E}-\mathrm{P}+$ & $2(0.8 \%)$ \\
\hline \multicolumn{2}{|l|}{ Chemotherapy, n (\%) } \\
\hline Adjuvant & $141(59.5 \%)$ \\
\hline Neoadjuvant & $96(40.5 \%)$ \\
\hline \multicolumn{2}{|l|}{ Anthracycline-based therapy, n (\%) } \\
\hline Yes & $199(83.9 \%)$ \\
\hline No & $38(16.1 \%)$ \\
\hline \multicolumn{2}{|c|}{ Cumulative dose of doxorubicin, $n$ ( $\%$ of anthracycline-based) } \\
\hline $240 \mathrm{mg} / \mathrm{m}^{2}$ & $171(85.9 \%)$ \\
\hline$<240 \mathrm{mg} / \mathrm{m}^{2}$ & $28(14.1 \%)$ \\
\hline \multicolumn{2}{|l|}{ Radiation, n (\%) } \\
\hline Yes & $109(46.2 \%)$ \\
\hline No & $128(53.8 \%)$ \\
\hline Concomitant radiation, $\mathrm{n}(\%)$ & $97(41 \%)$ \\
\hline
\end{tabular}

$\mathrm{m}^{2}$ (range $\left.120-240 \mathrm{mg} / \mathrm{m}^{2}\right) .96$ patients $(40.5 \%)$ received neoadjuvant and 141 (59.5\%) adjuvant trastuzumab. By the time of the analysis, 136 patients $(57.2 \%)$ had completed trastuzumab treatment as planned, $40(16.8 \%)$ were under trastuzumab therapy and 19 patients $(8.0 \%)$ had missed 1 or more trastuzumab administrations (absence for social reasons). 10 patients $(4.2 \%)$ discontinued trastuzumab due to disease recurrence during adjuvant treatment, and $1(0.04 \%)$ secondary to severe cutaneous rash. The median time of exposure was 8 months (range 2-12 months). Adjuvant radiotherapy was given to 109 patients $(46.2 \%)$; in 97 , this was administered concomitantly to trastuzumab. Radiotherapy to the left side chest wall was administered in 59 patients $(24.9 \%)$ and in 52 $(21.9 \%)$ left-sided radiotherapy was administered concomitant to trastuzumab.

\section{Cardiotoxicity}

A cardiotoxic event was found in 48 patients $(20.2 \%)$. Symptomatic heart failure occurred in 9 patients $(3.8 \%)$ and asymptomatic drop in LVEF occurred in 39 patients $(16.4 \%)$. No cardiac deaths were observed.

The median time to event occurrence was 4 months (range 2-11 months). Among patients who had a cardiac event, $64.5 \%$ (31 patients) discontinued trastuzumab. Discontinua- 
Table 2. Risk factors evaluated for association with trastuzumab cardiotoxicity

\begin{tabular}{lccl}
\hline Risk factor & Cardiotoxicity group & No cardiotoxicity group & p value multivariate \\
\hline Patients, $\mathrm{n}$ & 48 & 189 & \\
Cardiopathy, n (\%) & $5(10.4)$ & $9(4.7)$ & 0.073 \\
Hypertension, n (\%) & $14(29.2)$ & $58(30.6)$ & 0.198 \\
Diabetes mellitus, $\mathrm{n}(\%)$ & $2(4.1)$ & $19(10.0)$ & 0.099 \\
Anthracycline, n (\%) & $41(85.4)$ & $158(83.6)$ & 0.178 \\
Radiotherapy, n (\%) & $31(64.5)$ & $97(51.3)$ & 0.126 \\
Left-sided, $\mathrm{n}(\%)$ & $16(33.3)$ & $43(22.7)$ & 0.101 \\
Age $>55 \mathrm{years}, \mathrm{n}(\%)$ & $25(52.0)$ & $79(41.7)$ & 0.130 \\
BMI $>25 \mathrm{~kg} / \mathrm{m}^{2}, \mathrm{n}(\%)$ & $26(54.1 \%)$ & $97(51.3)$ & 0.236 \\
\hline
\end{tabular}

$\mathrm{BMI}=$ body mass index tion for non-cardiac reasons was found in $5 \%$ of the patients (1 patient with severe cutaneous rash and 10 patients with disease recurrence during trastuzumab therapy).

The median number of echocardiography assessments other than the baseline exam was 3 (range 0-6). In the cardiotoxicity group, the median number of assessments, including baseline, was 4 (range 2-6) and in the non-cardiotoxicity group, 3 (range 1-6). Median initial LVEF was $64.83 \pm 1.5 \%$ (no event) versus $64.81 \pm 1.5 \%$ (cardiac event group) $(\mathrm{p}=$ 0.26); median 3-month LVEF was $63.67 \pm 4 \%$ (no event) versus $52.12 \pm 3 \%$ (cardiac event group) $(\mathrm{p}=0.0036) .5$ patients $(2.1 \%)$ required hospitalization due to congestive symptoms.

\section{Risk Factors}

None of the potential cardiac risk factors assessed was associated with cardiotoxicity. The potential cardiac risk factors analyzed are shown in table 2 .

\section{Reversibility}

According to our definition, only 20 patients (41.6\%) recovered cardiac function after trastuzumab discontinuation, while 28 patients ( 6 patients with symptomatic and 22 with asymptomatic drop in LVEF) did not recover cardiac function. Among these 28 individuals, 7 (25\%) regained LVEF to a normal level ( $\geq 55 \%$ ), but below baseline assessment. The median time to LVEF recovery was 78 days (range 19-99 days).

Among the 48 patients who presented with cardiotoxicity, 23 initiated treatment with an antihypertensive drug. Previous use or initiation of antihypertensive drugs after the event was not associated with cardiac recovery $(\mathrm{p}=0.08)$. Among symptomatic patients $(\mathrm{n}=9)$, only 3 reported long-term symptoms associated with the cardiac event and 6 patients recovered to an asymptomatic state.

\section{Discussion}

Of our cohort of patients with early BC, $20.2 \%$ experienced a cardiac event. Nevertheless, symptomatic heart failure only occurred in $3.8 \%$. The majority of patients had no symptoms related to cardiac damage. Additionally, according to our criteria, $41.6 \%$ of patients recovered cardiac function. Of note, this was a non-selected patient population, which may reflect the actual profile and incidence of cardiac events seen in clinical practice.

These findings reflect a slightly higher rate of cardiac events than previously found in trastuzumab adjuvant clinical trials. Up to $4 \%$ of patients enrolled in these trials experienced severe cardiac heart failure, and up to $14 \%$ had asymptomatic decrease in LVEF, requiring discontinuation of trastuzumab [20]. In contrast to these results, in retrospective analyses with a 'real-world' population, Tarantini et al. [24] found $27 \%$ and Lamot et al. [25] 31\% of cardiotoxicity in patients receiving adjuvant trastuzumab. These values are similar to our data, highlighting that patient selection criteria may play an important role in the susceptibility for cardiac damage secondary to trastuzumab administration. Nevertheless, the fact that trials often have a quite heterogeneous definition of cardiotoxicity also has to be taken into account. Consequently, direct comparisons between clinical trials are extremely difficult.

Earlier studies identified several risk factors associated with a higher rate of cardiac events, including older age, higher body mass index, hypertension and use of anthracyclines [19-23]. In our analysis, potential risk factors were not associated with the development of trastuzumab-induced cardiotoxicity. Although not statistically significant, there was a higher rate of patients with previous cardiopathy in the cardiotoxicity group compared to patients with no cardiac events $(10.4$ vs. $4.7 \%, p=0.073)$. It is possible that if our cohort of patients available for analysis had been larger, we may have identified an association between cardiac risk factors and cardiac events rate. We recommend that patients with known cardiovascular risk factors and who are receiving anthracyclines should be carefully monitored, especially when treated subsequently with trastuzumab.

Guglin et al. [26] demonstrated that some medications used for hypertension treatment (ACE inhibitors and beta-blockers) may have a protective effect on the heart. Most hypertensive patients in our study were taking an antihypertensive agent, which could have provided some degree of heart protection. 
The median time to cardiac event development was 4 months. Others retrospective studies also demonstrated that cardiotoxicity was more frequent in the initial months of trastuzumab exposure $[24,25]$. In patients receiving trastuzumab, serum troponin, a potential marker for cardiac injury, was shown to be elevated in the first 3 cycles of treatment [27]. Thus, although troponin I levels are not currently indicated for monitoring $\mathrm{BC}$ treated with trastuzumab, these results suggest that patients should be more carefully monitored during the first months of trastuzumab use. The optimal surveillance for cardiotoxicity is not defined, but intensive serial monitoring of cardiac function at baseline, and at 3,6, and 9 months during treatment may be a reasonable choice [28].

In the present study, trastuzumab treatment was completed as planned by $57.2 \%$ of the patients. The high rate of patients not completing treatment as planned was a consequence of disease recurrence during (neo)adjuvant therapy (4.2\%), absence or delay in 1 or more administrations of the drug $(8 \%)$ and limiting dermatological toxicity $(0.04 \%)$. Of note, the major reason for the high rate of incomplete trastuzumab treatment was the fact that, at the time of our analysis, $16.8 \%$ of patients were still under trastuzumab therapy. Although $42.8 \%$ of the patients did not complete therapy as planned during the time of analysis, their LVEF had been monitored and could be analyzed for cardiotoxicity.

We observed that $13.1 \%$ (31) of patients discontinued trastuzumab due to cardiotoxicity; however, most patients were asymptomatic. Similarly, the NSABP B-31 trial showed that $15.5 \%$ of patients discontinued trastuzumab secondary to cardiac events [29]. In the HERA trial, the rate of symptomatic heart failure was $1.7 \%$ and asymptomatic decrease in LVEF was $7.1 \%$ [4]. The incidence of trastuzumab discontinuation due to cardiac disorders was low (4.3\%) [19]. This incidence rate was also seen in other trials, with asymptomatic declines in LVEF accounting for the vast majority of cardiotoxicity observed.

Trastuzumab-related cardiotoxicity is frequently considered a reversible event if the drug is discontinued. This was actually observed even in a patient population composed of metastatic BC patients at the M. D. Anderson Cancer Center. They reported that in patients with asymptomatic reduction in LVEF, cardiac function recovered completely in the great majority of patients, regardless of heart failure medication treatment [30]. This same cancer center group retrospectively reviewed patients who were referred to the department of cardiology. Most patients in this cohort had previously been exposed to anthracyclines. Even in this population, the LVEF returned toward baseline, with the mean LVEF increasing to $0.55 \pm 0.11[31]$.

The NSABP B-31 trial also demonstrated the reversibility of cardiac dysfunction secondary to trastuzumab use. In the
87 patients with asymptomatic drop in LVEF, only 15 still had an LVEF below 50\% [29]. Long-term analysis of patients included in the HERA trial, with a median follow-up of 3.6 years, showed that among the 73 patients with cardiac end points, $59(80.8 \%)$ reached recovery and only 6 presented progressive cardiac disease [32].

In the present study, we defined reversibility as the complete recovery of the LVEF to at least the baseline value as well as remission of related symptoms. This is a strict definition, which may have contributed to the low reversibility incidence found, namely $41.6 \%$. Some patients improved LVEF after treatment discontinuation, but in many LVEF did not return to previous baseline values, and thus this was not considered reversibility in our pre-established definition. Although assessments of LVEF by echocardiography were performed in the same institution using the same method of quantification, inter-observer variability and limitations intrinsic to the method could influence this finding and lead to an underestimation of the rate of reversibility.

The clinical impact of cardiac health for patients who still maintain some degree of cardiac dysfunction after trastuzumab discontinuation is unknown. Robust long-term data regarding cardiotoxicity after trastuzumab treatment are still lacking.

An important variable that also need to be considered is that the life expectancy for HER2+ BC patients is becoming increasingly longer. Patients can potentially be exposed to other cardiac risk factors during the course of their lives. Furthermore, although many patients recover their ventricular function after trastuzumab treatment, we do not have clear evidence that this will be sustained over the long term. Therefore, it may be important to follow patients even after the end of their adjuvant treatment. Strict control of other cardiac risk factors, especially in patients who require cardiac medication treatment, is strongly recommended.

In conclusion, we found that, in clinical practice, the incidence of trastuzumab-related cardiotoxicity was higher than the reported in adjuvant randomized controlled trials. Most patients presented with an asymptomatic drop in LVEF. We were not able to identify any risk factors for trastuzumab-induced cardiotoxicity. The reversibility of cardiac injury was not completely clear. Long-term maintenance of the quality of life and cardiac assessment of patients who remain disease free is required. The risk-versus-benefit profile of trastuzumab use remains tightly in favor of treatment, but cardiotoxicity should be carefully monitored.

\section{Disclosure Statement}

The authors declare no competing interests. 


\section{References}

1 Siegel R, DeSantis C, Virgo K, et al.: Cancer treatment and survivorship statistics, 2012. CA Cancer J Clin 2012;62:220-241.

2 Dahabreh IJ, Linardou H, Siannis F, et al.: Trastuzumab in the adjuvant treatment of earlystage breast cancer: A systematic review and metaanalysis of randomized controlled trials. Oncologist 2008;13:620-630.

3 Reese DM, Slamon DJ: HER-2/neu signal transduction in human breast and ovarian cancer. Stem Cells 1997;15:1-8.

4 Piccart-Gebhart MJ, Procter M, Leyland-Jones B, et al.: Trastuzumab after adjuvant chemotherapy in HER2-positive breast cancer. N Engl J Med 2005; 353:1659-1672.

5 Slamon D, Eiermann W, Robert N, et al.: Adjuvant trastuzumab in HER2-positive breast cancer. N Engl J Med 2011;365:1273-1283.

6 Harris CA, Ward RL, Dobbins TA, et al.: The efficacy of HER2-targeted agents in metastatic breast cancer: a meta-analysis. Ann Oncol 2011;22:13081317.

7 Perez EA, Romond EH, Suman VJ, et al.: Fouryear follow-up of trastuzumab plus adjuvan chemotherapy for operable human epidermal growth factor receptor 2-positive breast cancer: Joint analysis of data from NCCTG N9831 and NSABP B-31. J Clin Oncol 2011;29:3366-3373.

8 Joensuu H, Kellokumpu-Lehtinen PL, Bono P, et al.: Adjuvant docetaxel or vinorelbine with or without trastuzumab for breast cancer. N Engl J Med 2006;354:809-820.

9 Slamon DJ, Leyland-Jones B, Shak S, et al.: Use of chemotherapy plus a monoclonal antibody against HER2 for metastatic breast cancer that overexpresses HER2. N Engl J Med 2001;344:783-792.

10 Buzdar AU, Ibrahim NK, Francis D, et al. Significantly higher pathologic complete remission rate after neoadjuvant therapy with trastuzumab, paclitaxel, and epirubicin chemotherapy: Results of a randomized trial in human epidermal growth factor receptor 2-positive operable breast cancer. J Clin Oncol 2005;23:3676-3685.

-11 Buzdar AU, Valero V, Ibrahim NK, et al.: Neoadjuvant therapy with paclitaxel followed by 5-fluorouracil, epirubicin, and cyclophosphamide chemotherapy and concurrent trastuzumab in human epidermal growth factor receptor 2-positive operable breast cancer: An update of the initial randomized study population and data of additional patients treated with the same regimen. Clin Cancer Res 2007;13:228-233.
12 Untch M, Fasching PA, Konecny GE, et al.: Pathologic complete response after neoadjuvant chemotherapy plus trastuzumab predicts favorable survival in human epidermal growth factor receptor 2-overexpressing breast cancer: Results from the TECHNO trial of the AGO and GBG study groups. J Clin Oncol 2011;29:3351-3357.

13 Seidman A, Hudis C, Pierri MK, et al.: Cardiac dysfunction in the trastuzumab clinical trials experience. J Clin Oncol 2002;20:1215-1221.

14 Telli ML, Hunt SA, Carlson RW, et al.: Trastuzumab-related cardiotoxicity: Calling into question the concept of reversibility. J Clin Oncol 2007;25: 3525-3533.

15 Pugatsch T, Abedat S, Lotan C, et al.: Anti-erbB2 treatment induces cardiotoxicity by interfering with cell survival pathways. Breast Cancer Res 2006;8:R35.

16 Bria E, Cuppone F, Milella M, et al.: Trastuzumab cardiotoxicity: biological hypotheses and clinical open issues. Expert Opin Biol Ther 2008;8:19631971.

17 Erickson SL, O'Shea KS, Ghaboosi N, et al.: ErbB3 is required for normal cerebellar and cardiac development: A comparison with ErbB2-and heregulindeficient mice. Development 1997;124:4999-5011.

18 Sawyer DB, Zuppinger C, Miller TA, et al.: Modulation of anthracycline-induced myofibrillar disarray in rat ventricular myocytes by neuregulin1beta and anti-erbB2: Potential mechanism for trastuzumab-induced cardiotoxicity. Circulation 2002;105:1551-1554.

19 Suter TM, Procter M, van Veldhuisen DJ, et al.: Trastuzumab-associated cardiac adverse effects in the herceptin adjuvant trial. J Clin Oncol 2007;25: 3859-3865.

20 Tan-Chiu E, Yothers G, Romond E, et al.: Assessment of cardiac dysfunction in a randomized trial comparing doxorubicin and cyclophosphamide followed by paclitaxel, with or without trastuzumab as adjuvant therapy in node-positive, human epidermal growth factor receptor 2-overexpressing breast cancer: NSABP B-31. J Clin Oncol 2005;23: 7811-7819.

21 Pinder MC, Duan Z, Goodwin JS, et al.: Congestive heart failure in older women treated with adjuvant anthracycline chemotherapy for breast cancer. J Clin Oncol 2007;25:3808-3815.

22 Serrano C, Cortes J, De Mattos-Arruda L, et al.: Trastuzumab-related cardiotoxicity in the elderly: A role for cardiovascular risk factors. Ann Oncol 2012;23:897-902.
3 Gianni L, Salvatorelli E, Minotti G: Anthracycline cardiotoxicity in breast cancer patients: Synergism with trastuzumab and taxanes. Cardiovasc Toxicol 2007;7:67-71.

24 Tarantini L, Cioffi G, Gori S, et al.: Trastuzumab adjuvant chemotherapy and cardiotoxicity in realworld women with breast cancer. J Card Fail 2012; 18:113-119.

25 Lamot C, Rottey S, De Backer T, et al.: Cardiac toxicity of trastuzumab: experience at the Ghent Unversity Hospital, Belgium. Acta Clin Belg 2010; 65:300-304.

26 Guglin M, Hartlage G, Reynolds C, et al.: Trastuzumab-induced cardiomyopathy: Not as benign as it looks? A retrospective study. J Card Fail 2009;15:651-657.

27 Cardinale D, Colombo A, Torrisi R, et al.: Trastuzumab-induced cardiotoxicity: Clinical and prognostic implications of troponin I evaluation. J Clin Oncol 2010;28:3910-3916.

28 Jones AL, Barlow M, Barrett-Lee PJ, et al.: Management of cardiac health in trastuzumabtreated patients with breast cancer: updated United Kingdom National Cancer Research Institute recommendations for monitoring. Br J Cancer 2009; 100:684-692.

29 Romond EH, Jeong JH, Rastogi P, et al.: Sevenyear follow-up assessment of cardiac function in NSABP B-31, a randomized trial comparing doxorubicin and cyclophosphamide followed by paclitaxel (ACP) with ACP plus trastuzumab as adjuvant therapy for patients with node-positive, human epidermal growth factor receptor 2-positive breast cancer. J Clin Oncol 2012;30:3792-3799.

30 Guarneri V, Lenihan DJ, Valero V, et al.: Longterm cardiac tolerability of trastuzumab in metastatic breast cancer: the M.D. Anderson Cancer Center experience. J Clin Oncol 2006;24:4107-4115.

31 Ewer MS, Vooletich MT, Durand JB, et al. Reversibility of trastuzumab-related cardiotoxicity: New insights based on clinical course and response to medical treatment. J Clin Oncol 2005;23:78207826.

32 Procter M, Suter TM, de Azambuja E, et al. Longer-term assessment of trastuzumab-related cardiac adverse events in the Herceptin Adjuvant (HERA) trial. J Clin Oncol 2010;28:3422-3428. 